\title{
Development of an Information Preservation Method for Subsonic, Micro-Scale Gas Flows
}

\author{
Quanhua Sun, Iain D. Boyd, Jing Fan \\ Department of Aerospace Engineering \\ University of Michigan, Ann Arbor, MI, 48109, USA
}

\begin{abstract}
The development of an information preservation (IP) method is described in this paper. This effort is aimed at increasing our understanding of rarefied gas behavior of subsonic, micro-scale gas flows. The IP method preserves macroscopic information of the flow in the simulated particles. It applies conservation laws for binary collisions of the particles following the movement in the DSMC method, and updates the information using the Euler equations. The IP results exhibit very low levels of statistical scatter, which helps apply the method to low speed gas flows. A difficulty in implementing the IP method is also discussed.
\end{abstract}

\section{INTRODUCTION}

Micro-scale gas flows are the flows in and around micro-scale devices, such as Micro Air Vehicles (MAV) and micro-electro-mechanical systems (MEMS). Because of the very small length scale associated with these flows, the mean free path of the molecules can be comparable to the system size, and the flows are beyond the continuum regime. In these rarefied gas flows, the concepts of continuum dynamics are not valid. Methods based on a kinetic formulation must therefore be applied.

One of these methods is the direct simulation Monte Carlo (DSMC) method [1], which models a real gas as thousands or millions of discrete computational particles. In the last few years, DSMC method has been extensively used for hypersonic flows, and some researchers have already applied it to micro-channel flows. However, microchannel simulations present great challenges not encountered in previous DSMC applications [2]. First of all, the flow velocities are generally significantly less than the speed of sound. The governing equations for subsonic flows are elliptic. Hence, inflow or outflow conditions must be applied to all the boundaries. Secondly, it is difficult for DSMC to isolate the useful signal from "noise" in low speed flows. In order to reduce the statistical scatter, the total sample size must be greatly increased. Then few flows of practical interest can be simulated due to the limit of CPU time [3].

An alternative approach is the information preservation (IP) method, which is very effective in reducing the statistical scatter [4]. The IP method preserves macroscopic information as the particles simulated in DSMC move and interact with each other and domain boundaries. Recently, a 2D IP code was implemented and applied to a flow over a NACA 0012 airfoil [5] and a micro-channel flow [6]. In the implementation, the number density of cells and two velocity components of both particles and cells were preserved; also the isothermal assumption was used, which limits the application of the IP method.

The main objectives of the present investigation are, first, to present the further development of the information preservation method, and, second, to show the advantage of the IP method and a difficulty encountered to implement this technique.

\section{BASIC IDEA OF THE INFORMATION PRESERVATION METHOD}

The DSMC method is a particle simulation method based on kinetic theory. The real gas is modeled by a statistically representative set of simulated particles. It is known that each particle represents an enormous number of real molecules, and possesses random thermal properties according to certain distribution functions. Furthermore,

CP585, Rarefied Gas Dynamics: 22 ${ }^{\text {nd }}$ International Symposium, edited by T. J. Bartel and M. A. Gallis

(C) 2001 American Institute of Physics 0-7354-0025-3/01/ $\$ 18.00$

547 
those real molecules must have certain aggregate information, which is not subject to statistical fluctuation at the molecular level. In this meaning, the particle simulated in DSMC carries the aggregate information and the statistical scatter as a whole. The information preservation method aims to preserve the aggregate information of the real molecules, and to obtain the macroscopic quantities of interest through the statistical averaging of the information.

In the IP method, the particles follow the movement as in the DSMC method, which means the result of the DSMC method can still be obtained. When the particles move and interact with each other and the boundaries, the aggregate information carried by the particles (momentum and energy) changes. However, the information is conserved even for rarefied gas flows, except that care must be taken with viscosity and thermal conductivity. The effects of the viscosity and the thermal conductivity can be considered during the particle collisions and gas-surface interactions. Besides these effects, the information is updated according to the Euler equations. Then two steps at each time level are considered: the Lagrangian step and the Eulerian step. During the Lagrangian step, the information of particles that are selected to collide is adjusted according to general conservation laws. When particles cross cell edges or reflect from a wall, certain information transfer may happen. During the Eulerian step, the preserved information is updated using the Euler equations. At the end of each time level, the cell information is sampled since it is needed by the next time step. This cell information can also be used as an effective boundary condition.

\section{IMPLEMENTATION OF THE INFORMATION PRESERVATION METHOD}

In principle, any macroscopic information of the flow can be preserved by the simulated particles. For subsonic, micro-scale flows, non-equilibrium between the translational energy and internal energy is not significant. Then number density, velocity in two dimensions, and translational temperatures in three dimensions are enough to describe this kind of flow. In our 2D parallel IP code that is based on a parallel optimized DSMC code named "MONACO" [7], these variables are preserved as the macroscopic information. For subsonic flows, the variable soft sphere molecular model [1] is employed in the code.

The information for all the particles and cells is initialized by the ambient conditions after the computational domain is set up. Then two steps are executed in each time step. During the Lagrangian step, particles are selected to make pairs and binary collisions are performed between each pair. The momentum and energy conservation laws are used for the binary collisions. It is assumed that the preserved velocity and the preserved temperatures of two particles are the same after the collision. If new particles are injected into the computational domain, the ambient conditions or the local cell information is given to the new particles according to the boundary conditions. All particles are advanced according to their individual velocity for this time step. When a particle collides with a wall, the information of the particle is changed. If it is a specular reflection, the preserved temperatures of the particle are kept unchanged and the preserved velocity component perpendicular to the wall is reversed. When it is a diffuse wall, the preserved temperatures of the particle are accommodated to the wall temperature and the preserved velocity of the particle is set to the velocity of the wall. During the Eulerian step, the information of particles is updated and the cell information is sampled at the end of each time step.

The Euler equations used in the Eulerian step are modified for 2D non-equilibrium flows as follows:

$$
\begin{gathered}
\frac{d[1 / n]}{d t}=\frac{1}{n}\left[\frac{\partial u}{\partial x}+\frac{\partial v}{\partial y}\right] \\
\frac{d u}{d t}=-\frac{1}{n \cdot m} \frac{\partial p_{x}}{\partial x} \\
\frac{d v}{d t}=-\frac{1}{n \cdot m} \frac{\partial p_{y}}{\partial y} \\
\frac{d\left[\frac{1}{2} R \cdot T_{x}+\frac{u^{2}}{2}\right]}{d t}=-\frac{1}{n \cdot m} \frac{\partial\left[p_{x} \cdot u\right]}{\partial x} \\
d\left[\frac{1}{2} R \cdot T_{y}+\frac{v^{2}}{2}\right] \\
\frac{[t}{d t}=-\frac{1}{n \cdot m} \frac{\partial\left[p_{y} \cdot v\right]}{\partial y}
\end{gathered}
$$


In the above equations, $n$ is the number density, $m$ is the mass of molecules, $u$ and $v$ are the velocities, $T_{x}$ and $T_{y}$ are the temperatures, $p_{x}$ and $p_{y}$ are the pressures, $R$ is the specific gas constant, $\frac{d}{d t}$ and $\frac{\partial}{\partial t}$ are the full derivative and the partial derivative, respectively.

It is difficult to calculate the derivatives because only the information of discrete particles is preserved. The derivatives on the right hand side of Equations (1)-(5) are calculated from the cell values sampled from the particles, which are:

$$
\begin{gathered}
u_{c}=\sum_{j=1}^{N}\left(\frac{u_{j}}{N}\right), v_{c}=\sum_{j=1}^{N}\left(\frac{v_{j}}{N}\right) \\
p_{i, c}=\sum_{j=1}^{N}\left(\frac{n_{j} \cdot k \cdot T_{i, j}}{N}\right), i=x, y \\
T_{i, c}=\sum_{j=1}^{N}\left(\frac{T_{i, j}}{N}\right), i=x, y, z \\
\frac{1}{n_{c}}=\sum_{j=1}^{N}\left(\frac{1 / n_{j}}{N}\right) \\
\frac{\partial}{\partial l}=\frac{\oiint_{c e l l} \frac{\partial}{\partial l} d x d y}{\oiint_{c e l l} 1 \cdot d x d y}, l=x, y
\end{gathered}
$$

Here, the subscript $\mathrm{j}$ denotes the particle and the subscript $\mathrm{c}$ denotes the cell. $\mathrm{N}$ is the number of particles in the cell, and $\mathrm{k}$ is the Boltzmann constant. If $n$ on the right hand side of Equations (1)-(5) is replaced by the ratio of the real number of particles in the cell to the volume of the cell, the conservation properties can still be guaranteed. Notice that the concept of number density is not good for a single particle, so the number density of a particle takes the value of the number density for the cell at the end of each step.

\section{ADVANTAGES AND DISADVANTAGES OF THE IP METHOD}

The IP method preserves the information of the flow, which contains much less statistical scatter compared with the DSMC method for low speed flows. In DSMC, the statistical scatter comes directly from the thermal movement of particles. In IP, the thermal movement of particles causes scatter only at the information level. The statistical scatter of the information can not be larger than the variation of the information in the whole flow field. Hence, the IP method can greatly reduce the computational cost for low speed flows. The simulation of many practical microscale gas flows becomes possible. Because the cell information is sampled for each time step, the macroscopic values of the flow field are known at any time. This helps apply effective boundary conditions for the DSMC method, which improves the application of the DSMC method.

A disadvantage of the IP method is that it is difficult to model the translational energy flux. The energy flux through a cell edge or a wall is zero in equilibrium flows. However, for gas at rest, the average translational energy for particles crossing the edge from one side is $2 \mathrm{kT}$, which is different from $3 / 2 \mathrm{kT}$, the average translational energy for particles in the cell. This difference is important when calculating the heat flux from the wall or when the flow is a non-equilibrium flow. The difference comes because the translational energy flux of the part in the direction perpendicular to the wall or the edge is twice the flux of the part in the other directions in equilibrium flows, which is a direct result from the microscopic movement of the molecules. One possible way to include this difference in the IP method is to introduce additional translational energy flux in that direction. Notice that the translational energy flux from one side is balanced by the flux from the opposite side in equilibrium flows. The calculation of the additional energy flux is based on the difference between the temperature of the particle and the temperature of the cell in the direction perpendicular to the cell edge or the wall. The difficulty here is how to assign the additional energy to the particles. 
In our implementation, when a particle crosses a cell edge, it carries its information to the new cell. Also, an additional energy of $\frac{1}{2} k\left(T_{p}-T_{c}\right) \cdot w_{p}$ is transferred from the original cell to the new cell. Here, $T_{p}$ and $T_{c}$ are the temperature of the particle and the temperature of the cell in the direction perpendicular to the cell edge, respectively, and $w_{p}$ is the particle weight of the original cell. The additional energy is uniformly distributed over all particles in the cell at the end of that time step. Here, the additional energy is divided by the particle weight since each particle represents a large number of molecules. A similar treatment is applied when a particle interacts with a wall except that the additional energy of $\frac{1}{2} k\left(T_{w}-T_{p}\right) \cdot w_{p}$ is transferred from the wall to the cell. However, investigation shows that the translational energy flux across a cell edge or from a wall in this model is not sufficient. It may be that the treatment of the additional energy reduces the difference of the temperatures between particle and cell. Hence, the additional energy may be increased to $\frac{1}{2} k\left(T_{p}-T_{c}\right) \cdot w_{p} \cdot a$, where a is greater than unity. Then further study is needed to fix the value of a. For many subsonic, micro-scale gas flows, the temperature variation is not large, and then the effect here will not be important.

\section{RESULTS}

Two cases are used to demonstrate the information preservation method. One is the flow over a flat plate, which is used to show the usefulness of the IP method. The other is the flow between two parallel plates at different temperatures, which exhibits the difficulty with translational energy flux in the IP method.

\section{Flow over a Flat Plate}

Consider airflow over a flat plate. Near the leading edge of the plate, the Reynolds number based on the distance from the start point of the plate is small. Hence, the viscous effect is strong and the local Knudsen number in this region is very high. So a continuum flow model is not valid. The DSMC method and the IP method are used to simulate the flow.

The simulated flow conditions are selected so that the Knudsen number based on the length of the plate is 0.01 and the free stream Mach numbers are 0.8 and 0.1, respectively. The computational domain is shown in Fig. 1, which is divided into 5000 cells. The inflow condition is set to the ambient condition listed in Table 1 , where $\mathrm{Kn}_{\infty}$ is based on the length of the plate. The particles entering from the outflow boundary are given the local cell

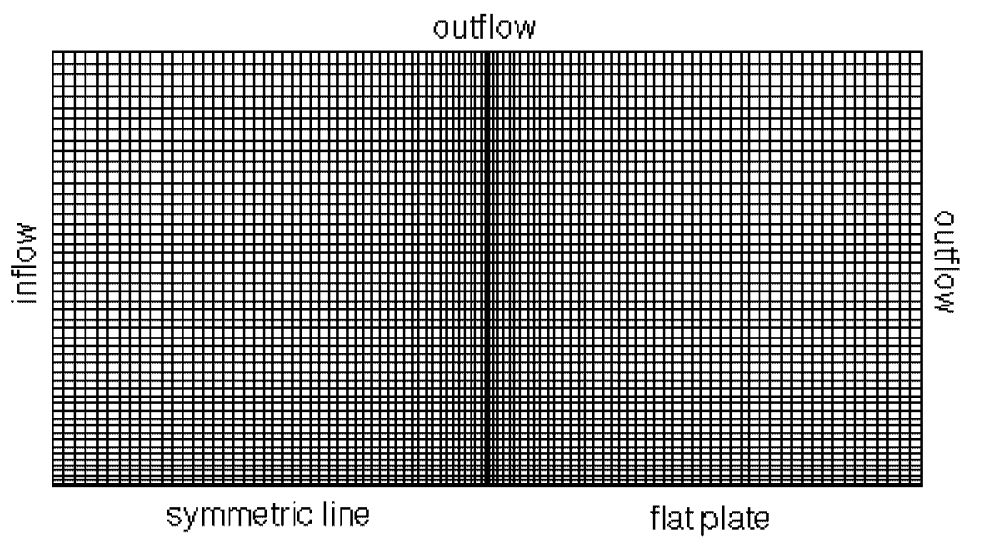

FIGURE 1. Computational Grid for Flow over a Flat Plate.

TABLE 1. Free Stream Conditions for Flow over a Flat Plate.

\begin{tabular}{ccccccc}
\hline Case & $\mathrm{L}(\mathrm{m})$ & $\mathrm{Ma}_{\infty}$ & $\rho_{\infty}\left(\mathrm{Kg} / \mathrm{m}^{3}\right)$ & $\mathrm{T}_{\infty}(\mathrm{K})$ & $\mathrm{U}_{\infty}(\mathrm{m} / \mathrm{s})$ & $\mathrm{Kn}_{\infty}$ \\
\hline $\mathrm{A}$ & $7.508 \times 10^{-6}$ & 0.8 & 0.8414 & 266.0 & 266.0 & 0.01 \\
$\mathrm{~B}$ & $5.584 \times 10^{-6}$ & 0.1 & 1.1314 & 299.4 & 35.28 & 0.01 \\
\hline
\end{tabular}


information. The time step is set to $5 \times 10^{-12} \mathrm{~s}$, which is smaller than the mean collision time of particles. During the simulation, about 35 particles are located in each cell. In order to reach the steady state, 20,000 iterations are executed before sampling the flow field.

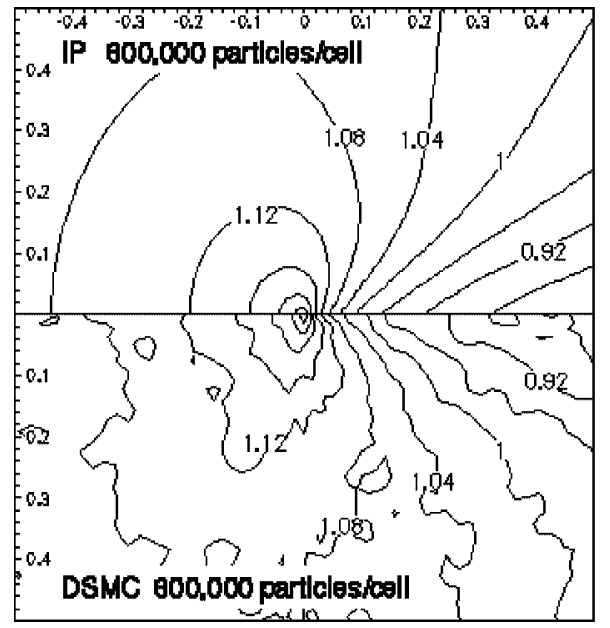

(a) density contours

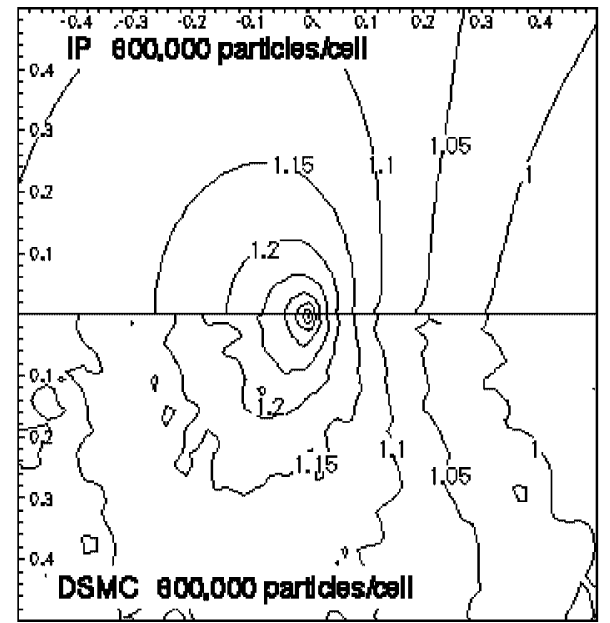

(b) pressure contours

FIGURE 2. Flow over a Flat Plate. $\mathrm{Ma}=0.8, \mathrm{Kn}=0.01$.

Figure 2 shows the density and pressure contours non-dimensionalized by the free stream conditions for Case A. The length here is non-dimensionalized by the length of the plate. Good agreement between the IP results and the DSMC results is obtained except that the DSMC results have some scatter with a total sample size of 800,000 particles per cell. The comparison of the pressure and the slip velocity on the plate between the DSMC results and the IP results is shown in Fig. 3. Again, the IP method predicts similar results to the DSMC method, which illustrates the utility of the information preservation method.
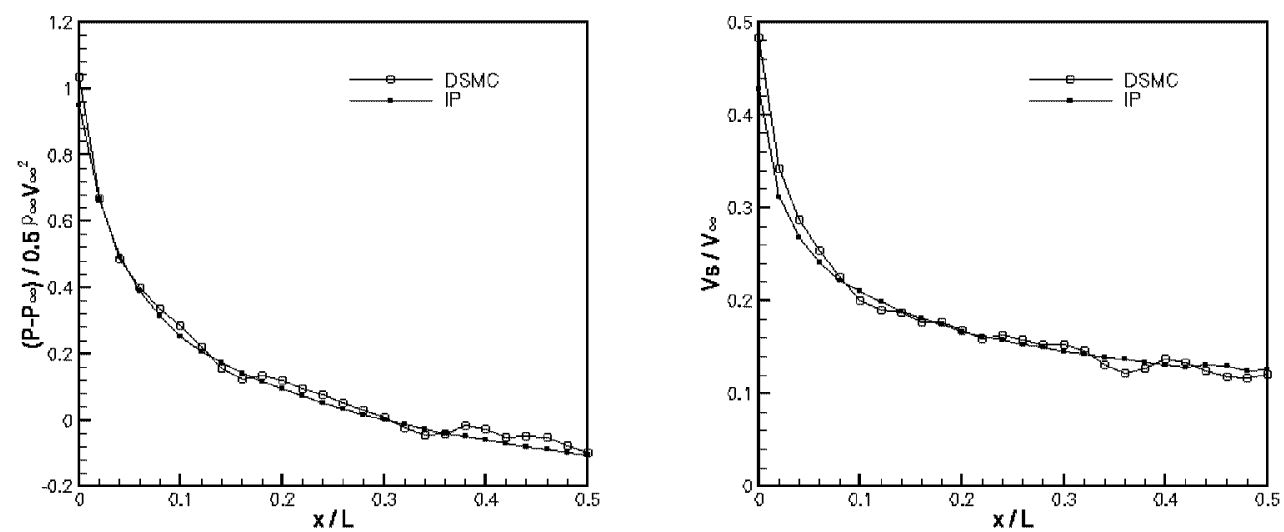

FIGURE 3. Comparison of Surface Properties for Flow over a Flat Plate.

Figure 4 shows the non-dimensional density contours for Case B. When the total sample size is 760,000 particles per cell, reasonable results are obtained by the IP method, while the large scatter in DSMC method makes it impossible to obtain meaningful results. Clearly, the IP method is able to simulate low speed flows.

\section{Flow between Two Plates at Different Temperatures}

Flow between two plates at different temperatures has been studied experimentally for different Knudsen numbers [8]. The Knudsen number is defined as the ratio of the mean free path of the molecules located at the centerline to the distance between the plates. The density in the experiment was set according to the Knudsen 


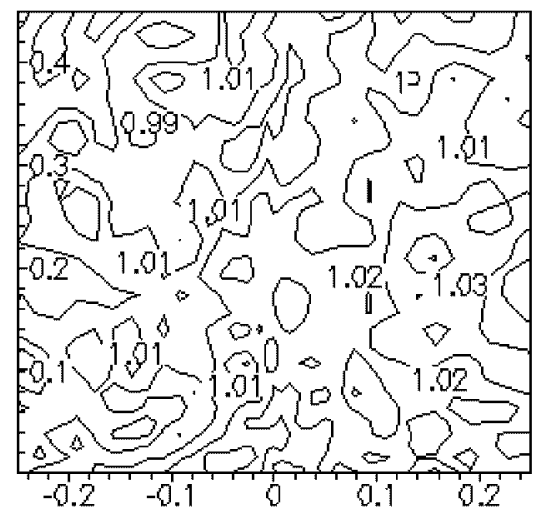

(a) DSMC

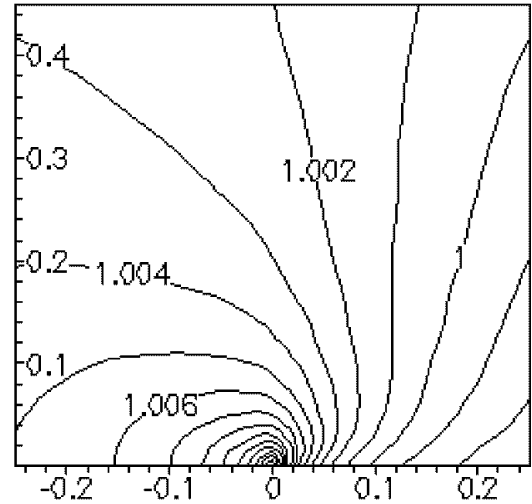

(b) IP

FIGURE 4. Density Contours for Flow over a Flat Plate. $\mathrm{Ma}=0.1, \mathrm{Kn}=0.01$.

number. Figure 5 shows the geometry used in the experiment. The distance between two 25.4-cm-long plates was $2.5 \mathrm{~cm}$. This is therefore approximately a one-dimensional problem. The temperature of the lower plate was kept at $288 \mathrm{~K}$, while the upper plate was at $368 \mathrm{~K}$. The argon gas between the plates was at a steady state condition during the experiment. The thermal accommodation coefficient was evaluated to be 0.826 using data obtained in the freemolecular regime.

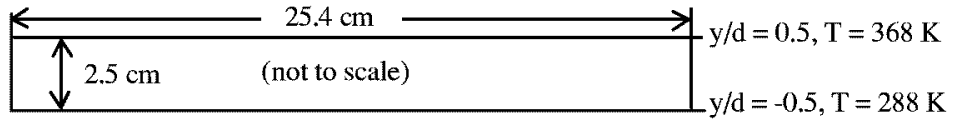

FIGURE 5. Parallel Plate Geometry.

In the simulation, 99 cells equally divided the whole regime between the plates $(300$ cells are used when $\mathrm{Kn}=$ 0.01 ). Thus, the cell size is less than the mean free path of particles. A total of 60,000 to 90,000 particles are simulated for all the cases. The time step is $1 \times 10^{-7} \mathrm{~s}$, which is smaller than the mean collision time for all cases. The results are obtained by sampling 2,000 time steps after the code is run for 20,000 time steps, which assumes a steady state is reached.

Figure 6(a) compares the simulated density from the IP method with the experimental data. When the Knudsen number is 0.01 , it is found that the IP results agree well with the DSMC results (not shown). It is clear that the agreement between the IP results and the experimental data decreases as the Knudsen number increases. This is because the model of the energy flux across the cell moves the flow towards equilibrium. Thus the IP result is close to the experimental data at a certain smaller Knudsen number. However, good agreement between the IP results and the theoretical solutions (uniform field) is obtained when the flow becomes free-molecular.

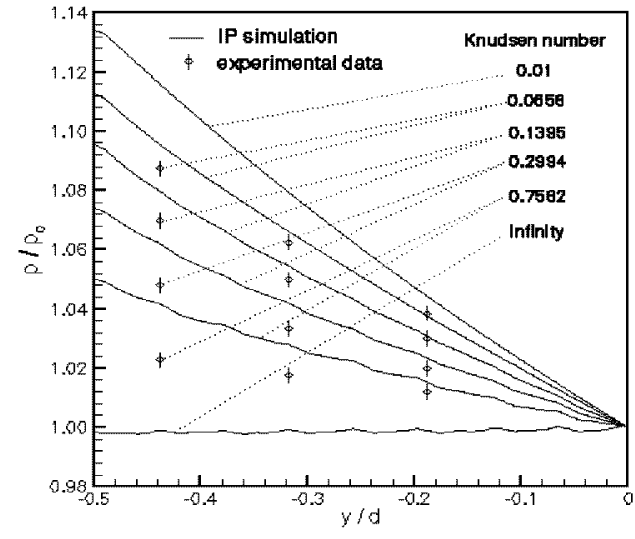

(a)

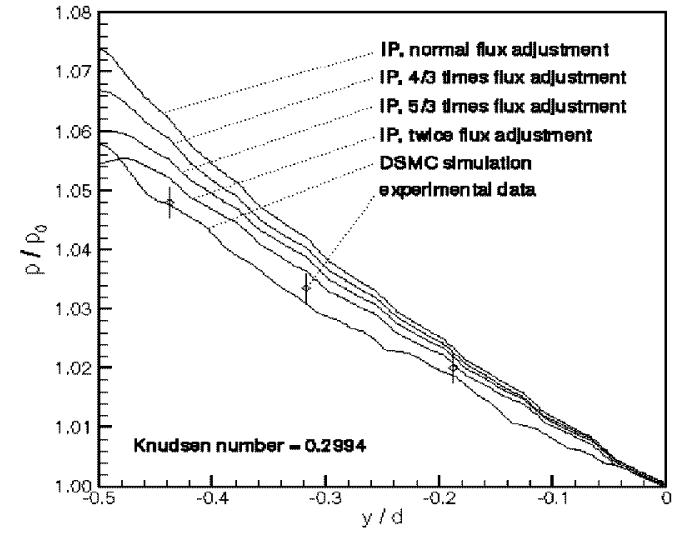

(b)

FIGURE 6. Density Profiles. 
An endeavor is made to account for the effect in the energy flux model of the IP method that occurs because the additional energy is equally shared by the particles. It is expected that additional energy flux is required to reduce this effect. Figure 6(b) shows the density distribution for several energy flux adjustments when the Knudsen number is 0.2994 . It is clear that increasing the energy flux can counter balance the effect to some degree. Investigation also finds that the energy flux adjustment depends on the local Knudsen number. Less energy flux adjustment is needed when the Knudsen number is higher. In Figure 6(b), notice that excess energy flux is introduced near the wall since the local Knudsen number is very high. Clearly, further work is required to formulate a physically accurate energy flux model for the IP method.

\section{CONCLUDING REMARKS}

Micro-scale gas flows are in general rarefied gas flows. The DSMC method can be used for this type of flow. However, large statistical scatter limits the application of the DSMC method when the speed of the flows is low. In this paper, the information preservation method was further developed for two-dimensional subsonic micro-scale gas flow.

The simulation results showed that the IP method worked effectively for the flows over a flat plate. It greatly reduced the statistical scatter, which enables the application of the particle methods. However, it is a challenge to model the translational energy flux across the cell. In our implementation, additional energy flux was required in order to obtain accurate results for high Knudsen number flows. For many subsonic micro-scale gas flows, temperature variation across the flow field is not large, and then the energy flux is not a large problem.

\section{ACKNOWLEDGMENTS}

The authors gratefully acknowledge support for this work from the Air Force Office of Scientific Research through MURI grant F49620-98-1-0433.

\section{REFERENCES}

1. Bird, G.A., "Molecular Gas Dynamics and the Direct Simulation of Gas Flows," Oxford, Clarendon, 1994

2. Nance, R.P., Hash, D.B., and Hassan, H.A., "Role of Boundary Conditions in Monte Carlo Simulation of MEMS Devices," AIAA Paper 97-0375, 1997.

3. Oh, C.K., Oran E.S., and Cybyk, B.Z., "Microchannel Flow Computed with the DSMG-MLG," AIAA Paper 95-2090, 1995

4. Fan, J., and Shen, C., "Statistical Simulation of Low-Speed Unidirectional flows in Transition Regime," in Proceedings of the 21th International Symposium on Rarefied Gas Dynamics, edited by R. Brum, et al., Marseille, 1998, pp. 245-252

5. Fan, J., Boyd, I.D., Cai, C.P., Hennighausen, K. and Candler, G.V., "Computation of Rarefied flows around a NACA 0012 Airfoil," AIAA Paper 99-3804, 1999

6. Cai, C.P., Boyd, I.D., Fan, J. and Candler, G.V., "Direct Simulation Method for Low-speed Micro-Channel-Flows," AIAA Paper 99-3801, 1999

7. Dietrich, S., and Boyd, I.D., Journal of Computational Physics, Volume 126, 328-342 (1996)

8. Teagan, W.P., and Springer, G.S., The Physics of Fluids, Volume 11, number 3, 497-506 (1968) 